\title{
Core body temperature is not a reliable parameter to follow the reproductive cycle in female marmoset monkey (Callithrix jacchus)
}

\author{
C. Drummer ${ }^{1}$, J. König ${ }^{2}$, K. Eildermann ${ }^{1}$, E. Fuchs ${ }^{2,3}$, R. Behr ${ }^{1}$, and K. Hoffmann ${ }^{2}$ \\ ${ }^{1}$ Stem Cell Biology Unit, German Primate Center, Göttingen, Germany \\ ${ }^{2}$ Clinical Neurobiology Laboratory, German Primate Center, Göttingen, Germany \\ ${ }^{3}$ Department of Neurology, University Medical Center Göttingen, Göttingen, Germany \\ Correspondence to: K. Hoffmann (kplassmann@t-online.de)
}

Received: 8 July 2014 - Revised: 4 September 2014 - Accepted: 8 September 2014 - Published: 23 October 2014

\begin{abstract}
Marmosets represent an attractive and widely used animal species in biomedical research, and the routine monitoring of female reproductive cycles is often mandatory in the fields of reproductive biology and stem cell research. Today, the established method for the reliable detection of ovulation is the determination of progesterone concentrations from blood samples. This method is based on relatively frequent handling and blood collections; therefore, less invasive alternatives would help to reduce stress on the animals. Here, we investigated whether the core body temperature of marmosets would show a correlation with cycle-dependent hormonal fluctuations, as has been described for humans and other primate species. In particular, the objective was to investigate whether the telemetric recording of core body temperature could replace progesterone measurements as a reliable, less invasive method for the detection of ovulation in these animals. Here we show that the core body temperature parameters in female marmosets were characterized by frequent variations, but they were not related to particular days or phases during the reproductive cycle. Therefore, the recording of core body temperature in our controlled standard experimental setting is not an appropriate method to monitor the reproductive cycle in female marmosets, and cannot replace serum progesterone measurement as a state-of-the-art method.
\end{abstract}

\section{Introduction}

Common marmoset monkeys (Callithrix jacchus) are small, diurnal nonhuman primates from South America; their body size of around $25 \mathrm{~cm}$ in length and a weight of $300-450 \mathrm{~g}$ enable easy handling procedures. Marmosets are social animals that can easily be housed in family groups, and breed well in controlled laboratory conditions with a high reproductive success showing no seasonal limitations. Therefore, marmosets represent an attractive and widely used species in different fields of biomedical research, including neurobiology, virology, toxicology, reproductive biology, and stem cell research (for review see Hearn, 1983; Mansfield, 2003; Orsi et al., 2011; 't Hart et al., 2012; Zühlke and Weinbauer, 2003).
Routine monitoring of the female reproductive cycle is often necessary, especially in the fields of reproductive biology and stem cell research. In marmosets, the ovarian cycle lasts around 28 days, with a follicular phase of about 8 days. But in common with most New World primates, marmosets do not menstruate or show other overt indications of the female cycle phase (Harding et al., 1982; Hearn, 1983; Mansfield, 2003). Today, the established method for reliable detection of the ovulation day is the determination of progesterone concentration from blood samples (Hanazawa et al., 2012; Harding et al., 1982). Although minimally invasive, this method is based on relatively frequent handling and blood collection, usually two times per week. Therefore, studies have been conducted for alternative determination of the timing of ovulation, such as the quantification of urinary estrogenic metabolites and fecal reproductive steroids or the 
analysis of vaginal swabs. The latter one was reported not to be a very sensitive single sampling indicator of the timing of ovulation (Gluckman et al., 2004).

Samples from feces and urine are sufficiently available and protocols for the determination of steroidal hormones are well established (French et al., 1996; Sousa and Ziegler, 1998). In feces, the determination of the periovulatory period can be roughly estimated with the increase of progesterone and estradiol (Ziegler and Sousa, 2002). However, one has to consider that after a surge the appearance of the steroids in feces is delayed for about $48 \mathrm{~h}$ as it was shown after injection of radiolabelled steroids (Shille et al., 1984; Ziegler et al., 1989). This delay is also observed when comparing the augmentation in fecal pregnandiol to the increase of plasma progesterone (Heistermann et al., 1993).

In urine samples, the peak of steroids is delayed only for 8-24 h (Ziegler et al., 1989) and their determination is less time-consuming than the analysis of fecal samples. Ovulation is detectable by determination of urinary pregnandiol-3glucuronide (PdG) from 3 consecutive days (Mustoe et al., 2012).

From this it is obvious that profiles of reproductive steroids from urinary and fecal samples allow only a retrospective determination of ovulation (Nievergelt and Pryce, 1996; Ziegler et al., 1996). For retrieval of early staged embryos (day 4-6), these approaches do not provide information about the day of ovulation with the same reliability and accuracy as the wellestablished assessment of blood progesterone concentrations (Hanazawa et al., 2012).

In women, a popular method for the monitoring of the reproductive cycle is the recording of basal body temperature, because core body temperature parameters vary with the different menstrual cycle phases. In a prospective field trial published in 1968, it was reported that ovulation takes place after a period of 6 days with a lower basal temperature, followed by a period of 3 days immediately after ovulation when body temperature rises for 0.2 to $0.4^{\circ} \mathrm{C}$ and stayed increased until menstrual bleeding occurs (Marshall, 1968, 1976; but see also Norris, 1952). This change in temperature is a result of alterations in the hormone profile throughout the reproductive cycle. Whereas estradiol lowers the core body temperature in the follicular phase, elevated circulating progesterone results in an increase in temperature in the luteal phase (Baker and Driver, 2007; Cagnacci et al., 1996, 1997, 2002; Coyne et al., 2000; Lee, 1988). In addition, the blunted nocturnal temperature decline during the luteal phase results in a decrease in amplitude of the temperature rhythm compared with the follicular phase (Baker and Driver 2007; Cagnacci et al., 1996, 1997; Coyne et al., 2000; Lee, 1988).

In nonhuman primates (NHPs), reproductive cycledependent variations in body temperature rhythms show species-dependent differences. The nocturnal subcutaneous temperature profile in chimpanzees (Pan troglodytes) and the circadian core body temperature rhythm of chacma baboons (Papio hamadryas ursinus) resemble the human temperature pattern with a clear periovulatory temperature drop and a strong correlation of temperature changes with fluctuating estrogen and progesterone concentrations (Graham et al., 1977; Nyakudya et al., 2012). Similar core body temperature curves were reported for rhesus monkeys (Macaca mulatta after treatment with Pregonal - a purified mixture of follicle stimulating and luteinizing hormone plus human chorionic gonadotropin (Balin and Wan, 1968). In contrast, the subcutaneous body temperature of female rhesus monkeys was not different between the follicular and the luteal phase. Instead, the authors found an increase in core body temperature 2-3 days before ovulation (Barger et al., 2008). These differences may be due to methodological reasons such as measurement of core body versus subcutaneous temperature registrations.

So far, the effect of hormonal changes on core body temperature parameters has not been studied in female marmosets. A recent long-term study of circadian rhythms revealed in a female marmoset a drop in core body temperature that occurred in a 4-week interval, indicating a possible relationship with cycle phases (Hoffmann et al., 2012). To obtain more information about this possible association, we performed in the present study long-term telemetric registrations of core body temperature simultaneously with established monitoring of the reproductive cycle by determining progesterone concentrations from blood samples.

Given the fact that temperature monitoring is not retrospective and marmosets do not have any obvious outward displays of ovulation, the objective of this study was to investigate whether the telemetric recording of core body temperature could replace progesterone measurements as a reliable, less invasive method for the real-time detection of ovulation in marmoset monkeys.

\section{Materials and methods}

All experiments were performed in accordance with the European Communities Council Directive 86/609/EEC and the German Animal Welfare Act and were approved by the Lower Saxony Federal State Office for Consumer Protection and Food Safety, Oldenburg, Germany (AZ 33.9-42502-0411/0395).

\subsection{Animals}

Six adult female common marmoset monkeys (age 2631 months) were obtained from the breeding colony of the German Primate Center (Göttingen, Germany). The animals were pair-housed with castrated males in a temperature $\left(25 \pm 1^{\circ} \mathrm{C}\right)$ and humidity-controlled $(65 \pm 5 \%)$ facility. These parameters were controlled daily. Room air was changed approximately 10 times per hour and filtered adequately. Illumination was provided by artificial lighting on a $12.5: 11.5 \mathrm{~h}$ light: dark cycle with dusk and dawn effects for the first and last $30 \mathrm{~min}$ of the light phase. Each cage 
$(w \times d \times h=66 \times 80 \times 150 \mathrm{~cm}$, Ebeco, Castrop-Rauxel, Germany) was furnished with wooden branches, and shelves and contained a wooden sleeping box $(24 \times 21 \times 18 \mathrm{~cm})$. The floor under the cages was covered by paper sheets. Urine, feces and leftovers were removed daily by exchange of the paper sheets. The study room and the cages were cleaned in weekly intervals and disinfected with water and Biguacid (Antiseptica, Pohlheim/Brauweiler, Austria).

The marmosets were fed ad libitum with a pellet diet (ssniff Spezialdiäten, Soest, Germany). In addition, $20 \mathrm{~g}$ mash per animal were served in the morning, and $30 \mathrm{~g}$ cleanly cut fruits or vegetables mixed with noodles or rice were supplied in the afternoon. Drinking water was always available. All materials were changed regularly, cleaned and sterilized.

\subsection{Core body temperature}

To record core body temperature, animals were implanted with Remo 200 radiotelemetry transmitters (Remo Technologies Ltd., Salisbury, UK). The implantation was performed under sterile conditions, and general anesthesia was induced by intramuscular injections (i.m.) of diazepam (0.3 mg kg${ }^{-1}$, Diazepam ${ }^{\circledR}$, Ratiopharm, Germany), alfaxolone $\left(10 \mathrm{mg} \mathrm{kg}^{-1}\right.$, Alfaxan ${ }^{\circledR}$, Vetoquinol, UK) and glycopyrronium bromide $\left(0.01 \mathrm{mg}\right.$ per animal, Robinul ${ }^{\circledR}$, Riemser, Germany). Effective depth of anesthesia was confirmed repeatedly by checking for an absent pedal withdrawal reflex, and body temperature was maintained at $38^{\circ} \mathrm{C}$ using a heating pad (Horn, Gottmadingen, Germany). After the induction of anesthesia, the animals were placed in dorsal spine position and shaved in the umbilical region. A $2 \mathrm{~cm}$ abdominal midline incision was made to open the peritoneal cavity. The sterile Remo 200 transmitter was inserted and fixed to the abdominal wall with a 4-0 nonabsorbable Mersilene suture (Ethicon, Germany); the muscle layer was closed with the same suture. Subsequently, the skin was closed with a 5-0 absorbable Safil ${ }^{\circledR}$ suture (Braun, Germany).

The animals were monitored until they awoke from anesthesia and were then returned to their home cages. They received i.m. injections of Meloxicam $\left(0.5 \mathrm{mg} \mathrm{kg}^{-1}\right.$, Metacam ${ }^{\circledR}$, Boehringer Ingelheim, Germany) approximately 20 min prior to and for 2 days following surgery, and prophylactic injections of the antibiotic amoxicillin $\left(60 \mathrm{mg} \mathrm{kg}^{-1}\right.$ i.m., Baytril ${ }^{\circledR}$, Bayer, Germany) were given three times every $48 \mathrm{~h}$.

Core body temperature recording began 1 week after surgery and was continued until the transmitter batteries were dead ( $\cong 3$ to $\cong 10$ months after implantation). The transmitter sent a temperature-dependent frequency modulated signal at $10 \mathrm{~s}$ intervals to a receiver near the cage. The signals were transferred to a personal computer-based acquisition program (eDaqc Version 1.7x, EMMS, Bordon, UK) and saved continuously from 13:20 to 13:00 LT the following day. The $20 \mathrm{~min}$ pause in recording allowed for saving and backing up the recorded data. For further analysis, data were imported to Microsoft Excel 2007 and GraphPad Prism software 4.0 (GraphPad Software Inc., San Diego, CA, USA) and averaged to $1 \mathrm{~min}$ bins.

To exclude a possible impact of blood sampling and handling on the core body temperature, determination of progesterone levels and ultrasonography were stopped after six complete cycles in two animals with a regular ovarian cycle, while registrations of core body temperature were continued for another 3-4 cycles.

At the end of the study, the telemetry transmitters were explanted according to the operative protocol described above, and the animals were returned to the breeding colony of the German Primate Center.

\subsection{Hormone analysis}

Measurement of progesterone started after a 2-week recovery phase from implantation of the radio transmitter. Blood samples with a volume of approximately $0.2 \mathrm{~mL}$ were taken from the venalarteria femoralis with a heparinized syringe twice a week from the trained vigilant animals. After blood collection, animals were held for 5-7 min with pressure on the puncture wound before recording their weight; as a reward they received about $1 \mathrm{~mL}$ gum arabic solution and were returned to their male partners. Samples were centrifuged ( $4000 \mathrm{~g}$ at $4{ }^{\circ} \mathrm{C}$ for $20 \mathrm{~min}$ ) and processed immediately after collection. The supernatant serum was used in the progesterone enzyme immunoassay using an antiserum raised in sheep against progesterone-11-hemisuccinate-bovine serum albumin (BSA) as described by Heistermann et al. (1993).

\subsection{Ultrasonography}

The ovarian reproductive cycle, particularly the development of follicles and corpora lutea, was monitored once a week by ultrasonography using a portable LOGIQ-e ultrasound scanner (GE Healthcare, Little Chalfont, Buckinghamshire, UK). The animals were familiar to the procedure and held recumbent by trained staff to give the examiner free access to the ventral abdominal wall. After ultrasonography the animals were rewarded with about $1 \mathrm{~mL}$ gum arabic solution. Ultrasonographic results of both ovaries were recorded. The functional structures could be separated by the differential echo of the tissues: round, dark gray and black (low echo) structures were known to be follicles while round, light gray and well-defined tissues (high echo) were identified as corpora lutea (see Figs. A6 and A7 in the Appendix).

\subsection{Data analysis and statistics}

Based on the $1 \mathrm{~min}$ averaged temperature data, the area under the curve (AUC; baseline set at $0^{\circ} \mathrm{C}$ ) for the dark phase of housing (19:00-06:30) was calculated using GraphPad prism software. Data sets with missing temperature recordings in the first or last $20 \mathrm{~min}$ of the dark phase were excluded from 


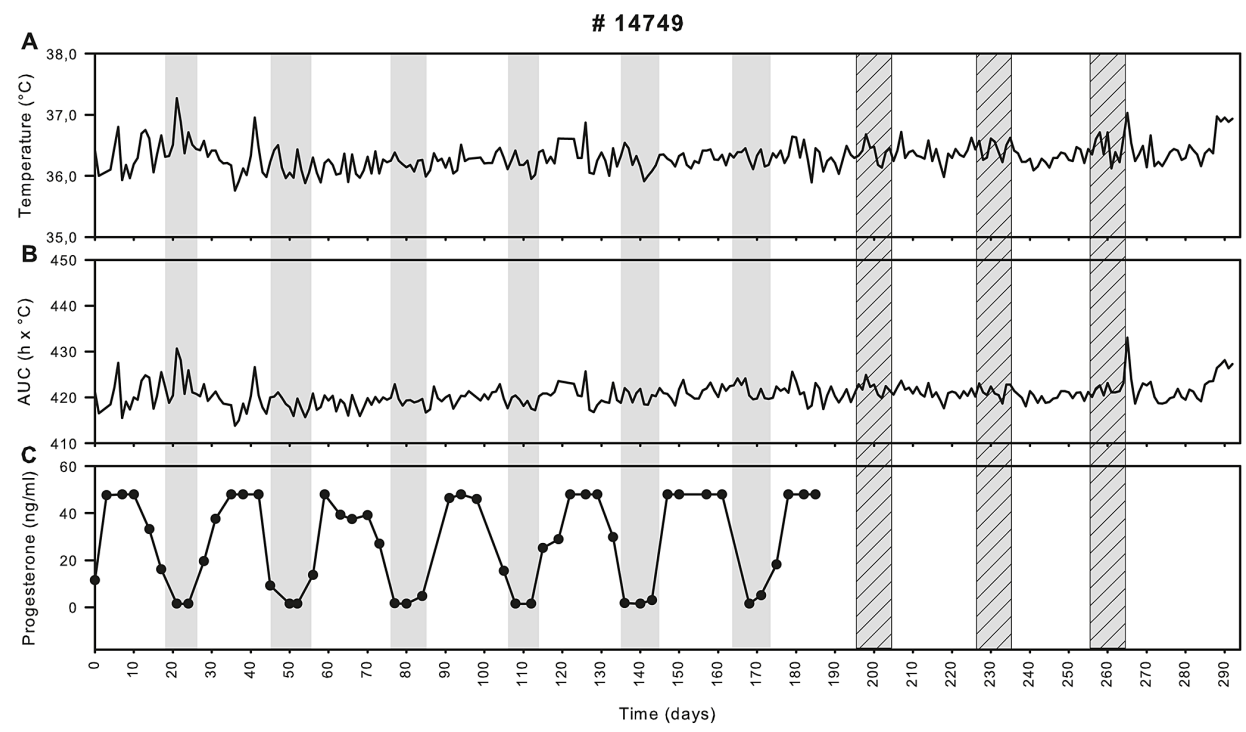

Figure 1. Diurnal variations in (a) minimal daily temperature $\left(T_{\min }\right)$, (b) area under the curve (AUC) of nocturnal body temperature, and (c) serum progesterone concentrations for the complete study period (six progesterone data-based cycles followed by three extrapolated cycles) for a representative, regular cycling female marmoset (\#14749). Follicular phases are highlighted in gray and hatched boxes indicate the follicular phases of extrapolated cycles.

AUC analyses (e.g., see Fig. A3). For determination of the minimum daily temperature $\left(T_{\min }\right)$, a curve fitting for the dark phase was performed using a polynomial fourth-order equation $\left(y=A+B x+C x^{2}+D x^{3}+E x^{4}\right)$, and the minimum of this curve was calculated according to Schmelting et al. (2014).

The follicular and the luteal phases of the ovarian cycle were identified based on the progesterone concentrations as described by Harding et al. (1982). Values between 1.5 and $10 \mathrm{ng} \mathrm{mL}^{-1}$ were assigned to the follicular phase while values above that were assigned to the luteal phase (Harlow et al., 1983). This was confirmed by results from the ultrasound scanning of the ovaries.

In addition, we investigated core body temperature in two females in 3-4 subsequent cycles without blood sampling or ultrasonography. These cycle phases were estimated based on the previously recorded regular cycle lengths in the same animals. This control phase was performed to exclude possible changes in core body temperature parameters caused by the experimental manipulations that could interfere with hormonal-related changes throughout the ovarian cycle.

The means for $T_{\min }$ and AUC for the follicular and luteal phases were calculated and compared using a paired samples $t$ test when normally distributed, or using the nonparametric Wilcoxon signed rank test when not normally distributed. Differences were considered statistically significant at the $95 \%$ confidence level $(p<0.05)$. All results are presented as the mean \pm standard error of the mean (SEM).

\section{Results}

The number of blood samples analyzed ranged from 30 to 52 per animal, covering an observation period between 15 and 26 weeks corresponding to 3-6 reproductive cycles. The progesterone concentration varied between $<1.5 \mathrm{ng} \mathrm{mL}^{-1}$ and $>48 \mathrm{ng} \mathrm{mL}^{-1}$.

Based on progesterone concentrations four out of the six animals investigated in this study were found to have regular reproduction cycles with a length of $27.4 \pm 1.6$ days consisting of a follicular phase of 7-10 days followed by a luteal phase ranging from 14 to 19 days (see Fig. 1, Figs. A1, A2 and $\mathrm{A} 3$ in the Appendix). In total, 20 cycles (3-6 per animal; see Table 1 for details) were included in the study. Ultrasonography findings corresponded with the progesterone concentration in $71.2 \%$ of cycles.

The remaining two animals were found to have irregular reproductive cycles, with abnormally long duration and/or constantly elevated progesterone concentrations, and were therefore excluded from further analysis (see Figs. A4 and A5 in the Appendix).

Figure 1 shows the daily results for $T_{\min }$, area under the curve (AUC), and progesterone concentrations for a representative animal (\#14749) for the complete study period, consisting of 6 cycles identified by progesterone concentrations and ultrasonography followed by 3 cycles calculated based on the length of previously recorded cycles.

Figure 2 shows the $24 \mathrm{~h}$ core body temperature rhythm of animal \#14749 for the luteal and the follicular phase of the reproductive cycle. It is characterized by a pronounced circadian variation with clear temperature differences of about 
Table 1. Minimal daily core body temperature $\left(T_{\min }\right)$ and area under the curve (AUC) of nocturnal body temperature values for follicular and luteal phases of the ovarian cycle. Data are given as the mean \pm SEM. The results for calculated cycles, not confirmed by progesterone concentrations, are in parentheses. No significant differences were found between the luteal and follicular phases.

\begin{tabular}{lrll}
\hline & \multicolumn{3}{c}{ Minimal daily temperature $\left({ }^{\circ} \mathrm{C}\right)$} \\
\hline Animal \# & No. of cycles & Follicular phase & Luteal phase \\
\hline 14749 & $6(3)$ & $36.28 \pm 0.07(36.39 \pm 0.03)$ & $36.28 \pm 0.02(36.35 \pm 0.02)$ \\
14745 & $6(3)$ & $35.93 \pm 0.02(35.93 \pm 0.04)$ & $35.92 \pm 0.02(35.87 \pm 0.02)$ \\
14687 & 5 & $35.78 \pm 0.03$ & $36.08 \pm 0.27$ \\
14963 & 3 & $35.90 \pm 0.10$ & $36.01 \pm 0.02$ \\
\hline \multicolumn{4}{c}{ Area under the curve of nocturnal temperature $\left(h \times{ }^{\circ} \mathrm{C}\right)$} \\
\hline Animal \# & No. of cycles & Follicular phase & Luteal phase \\
\hline 14749 & $6(3)$ & $420.33 \pm 0.71(421.05 \pm 0.01)$ & $420.36 \pm 0.38(421.12 \pm 0.42)$ \\
14745 & $6(3)$ & $416.29 \pm 0.55(415.98 \pm 0.49)$ & $416.92 \pm 0.22(415.11 \pm 0.29)$ \\
14687 & 5 & $414.62 \pm 0.23$ & $415.15 \pm 0.64$ \\
14963 & 3 & $414.43 \pm 1.51$ & $416.57 \pm 0.85$ \\
\hline
\end{tabular}

$2{ }^{\circ} \mathrm{C}$ between light and dark period. In the last $2 \mathrm{~h}$ of the light phase, the temperature drops from daytime values in the range of $38.5^{\circ} \mathrm{C}$ to nighttime values in the range of $36.5^{\circ} \mathrm{C}$ with lowest temperatures in the second half of the night.

Detailed analysis of core body temperature parameters during the reproductive cycle showed frequent variations for $T_{\min }$ within the range of 35.76 and $37.27^{\circ} \mathrm{C}$ and for AUC within 413.83 and $433.08 \mathrm{~h} \times{ }^{\circ} \mathrm{C}$. However, these variations were not associated with particular days or phases during the ovarian cycle. In addition, the animals investigated in this study showed no significant differences in the mean $T_{\min }$ between follicular $\left(35.97 \pm 0.12^{\circ} \mathrm{C}\right)$ and luteal $\left(36.07 \pm 0.09^{\circ} \mathrm{C}\right)$ phases. Likewise, the mean AUC for the nocturnal core body temperature was not significantly different between follicular $\left(416.42 \pm 1.58 \mathrm{~h} \times{ }^{\circ} \mathrm{C}\right)$ and luteal $\left(417.22 \pm 1.25 \mathrm{~h} \times{ }^{\circ} \mathrm{C}\right)$ phases. Results for the $T_{\min }$ and AUC for individual animals are summarized in Table 1.

Experimental manipulations had no detectable effect on core body temperature parameters. Follicular and luteal phase values were not significantly different in cycles with and without blood sampling or ultrasonography (Table 1).

\section{Discussion}

In this study, we investigated in adult pair-housed female marmosets possible relationships between fluctuations in hormone concentrations and the core body temperature registered via radiotelemetry transmitters implanted in the abdominal cavity. Simultaneously, the reproductive cycle was monitored using the well-established method of measuring serum progesterone levels (Harding et al., 1982) and ultrasonography of the ovaries.

Throughout the reproductive cycle low progesterone levels of $<10 \mathrm{ng} \mathrm{mL}^{-1}$ can be observed in the follicular phase, and high levels of $>10 \mathrm{ng} \mathrm{mL}^{-1}$ are characteristic of the luteal phase. The day before the progesterone concentration rises above $10 \mathrm{ng} \mathrm{mL}^{-1}$ is considered to be the day of ovulation (Harding et al., 1982; Harlow et al., 1983, 1984). In the present study, the cyclic variation of progesterone levels showed that only four out of the six investigated female marmosets had a regular reproductive cycle, and only those animals were included in further analyses. In these animals the mean cycle length of $27.4 \pm 1.6$ days was in the same range reported by other studies (Harding et al., 1982; Hearn, 1983; Nievergelt and Pryce, 1996). Similar to our findings irregular estrus cycles were reported in three out of six marmosets in a study investigating vaginal cytologic changes under the influence of circulating plasma progesterone (Gluckman et al., 2004). Possible effects on the reproduction cycle such as endocrine suppression due to a close genetic relationship to other animals in the same room, age, housing conditions or stress were eliminated by the study design as all animals were housed under identical conditions. The mean age was also not significantly different between animals with regular and irregular cycles ( $t$ test: $p=0.3 ; 29.5$ to 32 months at the start of the experiment). At the beginning of the study none of the monkeys was included in a breeding program. It is important to mention that the animals with irregular cycles proved their fertility in subsequent studies indicating that erratic cycles are not necessarily associated with infertility.

In women, variations of the core body temperature in different phases of the menstrual cycle are well described (Baker et al., 2001; Cagnacci et al., 2002, 1996; Coyne et al., 2000). Independent of the method for temperature measurement, several studies agree that the median and minimal daily body temperature are about $0.4{ }^{\circ} \mathrm{C}$ higher in the luteal phase than in the follicular phase, and that the amplitude of the diurnal temperature rhythm is reduced in the luteal phase. This effect is primarily a result of a blunted nocturnal temperature decline, resulting in an increased minimal temperature and a 
\# 14749

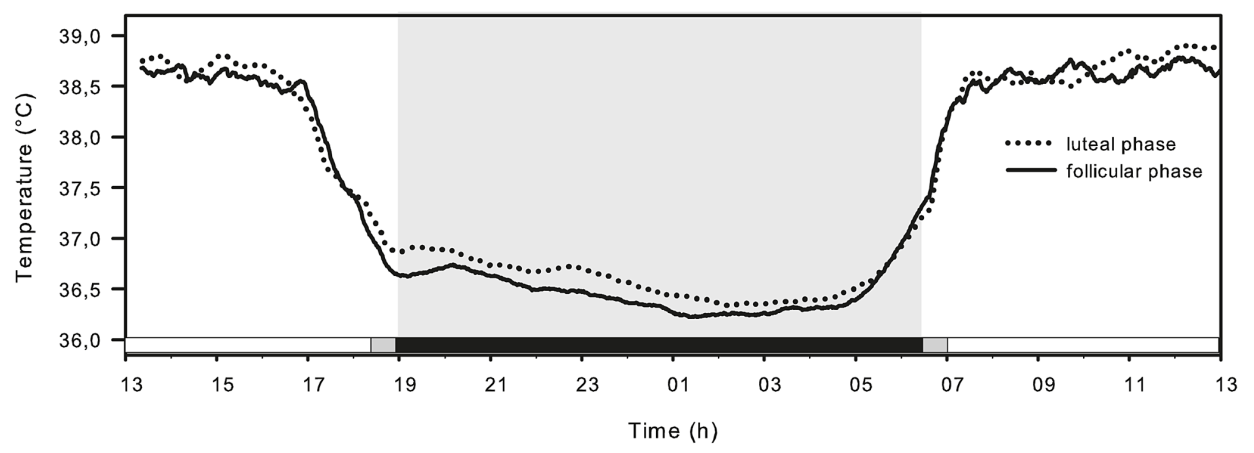

Figure 2. Core body temperature rhythm of animal \#14749 for the luteal (dotted line) and the follicular phase (solid line) of the reproductive cycle. The $24 \mathrm{~h}$ pattern is characterized by a clear circadian variation with pronounced temperature differences of about $2{ }^{\circ} \mathrm{C}$ between light and dark period (gray) with no significant differences between luteal and follicular phase. Temperature rapidly decreases in the last $2 \mathrm{~h}$ of the light phase from about $38.5^{\circ} \mathrm{C}$ to nighttime values in the range of $36.5^{\circ} \mathrm{C}$ and starts to increase to daytime values about $2 \mathrm{~h}$ before lights-on.

reduction in amplitude in the luteal phase (Baker et al., 2001; Cagnacci et al., 2002, 1996; Coyne et al., 2000; Lee, 1988). However, some authors also report an increase in the peak body temperature (Baker et al., 2001; Lee, 1988). These effects are considered to be mediated by the changes in serum progesterone concentrations (Baker and Driver, 2007; Hampl et al., 2006), or alternatively by a change in the progesteroneto-estrogen ratio (Cagnacci et al., 1997; Forman et al., 1987). The possible effects of the menstrual cycle on the phase of the diurnal temperature rhythm are not as clear. While Cagnacci et al. $(2002,1997,1996)$ were able to detect a delay of the temperature rhythm in the luteal phase, others found no difference (Baker et al., 2001; Coyne et al., 2000).

Taken together, the menstrual phase differences in core body temperature rhythm and peak body temperature in women are contradictory, while mean and minimum daily temperature provide reliable and reproducible readouts for hormone-dependent temperature changes, with a most pronounced variation in the minimal temperature during the night. Therefore, we focused on analyzing the minimal daily temperature and the nightly temperature AUC of the marmosets.

In contrast to the situation in women, we were not able to detect in marmosets any significant temperature differences between the follicular and luteal phase. This finding is consistent with earlier reports in rhesus monkeys, chacma baboons and chimpanzees. Notwithstanding, a clear periovulatory rise in core body temperature level enables the detection of ovulation in these three species, (Barger et al., 2008; Graham et al., 1977; Nyakudya et al., 2012). In marmosets, however, we could not detect a periovulatory temperature increase. At the current stage we cannot completely exclude the possibility that a periovulatory rise in core body temperature might also exist in marmoset monkeys. One may speculate that the pronounced day-night temperature variation masks a periovulatory rise which could not be detected by telemetric system used in this study.

It is known that marmosets (Petry and Maier, 1990) and, e.g., rats (Eikelboom, 1986; Poole and Stephenson, 1977) show a temperature elevation in response to handling procedures, and that experimental manipulations - for example, rectal temperature measurement or the transportation to a different room - can result in hyperthermia that can be detected for more than $60 \mathrm{~min}$ after the onset of the disturbance. Importantly, as shown in rats this hyperthermia can be reduced by habituation to the experimental procedure (Dallmann et al., 2006). To exclude such impact on the nightly core body temperature, all our experimental procedures were performed during the morning and the animals were well habituated to both the blood sampling twice a week and the weekly ultrasound investigations. To further exclude the impact of handling on core body temperature, we continued temperature recordings in two animals showing regular estrous cycles (\#14745 and \#14749). In these animals we omitted experimental manipulations such as blood sampling and ultrasound investigations and found no differences in core body temperature and AUC compared to the handling period (Figs. 1 and A1 in the Appendix). Therefore, a masking of a periovulatory rise in body temperature caused by our experimental procedure appears unlikely.

In marmosets, the pronounced day-night differences in core body temperature might be the result of a low thermoregulatory tolerance which was described in detail by Petry and Maier (1990). Even small changes or disturbances or changes in energy intake can have a noticeable impact on the diurnal body temperature rhythm. In the present study we did not monitor the animals' food intake and therefore we cannot exclude the possibility that days with lower minimal core body temperature corresponded with days of reduced energy intake. 
To avoid pregnancies during the monitoring of reproductive cycles, the female marmosets were housed in pairs with a castrated male. Surgical castration results in a significant decline in circulating testosterone (Nunes et al., 2002) and may act primarily via testosterone-dependent peripheral mechanisms resulting in reduced sexual activity as shown for castrated macaques (Michael and Zumpe, 1993; Zumpe et al., 1992). In the present study we did not monitor the behavior of the males. Consequently we cannot exclude the possibility that they exhibited reduced sexual activity.

Male as well as female marmosets possess scent glands and regularly show scent-marking behavior. It is well established that their communication system relies heavily on these olfactory signals which contain among others information about sex, hormonal status and identity (Epple, 1974). In males, the production of the chemical signals is under the control of testosterone. Orchectomy in the greater galago
(Galago crassicaudatus crassicaudatus) and in tree shrews (Tupaia belangeri) significantly diminishes marking activities and results in atrophic scent glands as well in structural changes of the glans penis (Dixson, 1976; von Holst and Eichmann, 1998). Similar effects with a strong impact on sexual behavior cannot be excluded for gonadectomized marmosets as sex and gonadal steroids play a central role in mammalian pheromonal communication (Baum and Bakker, 2013). Thus, the use of vasectomized males instead of castrated ones might be an additional option for future studies.

In summary, we found that although the determination of progesterone from blood serum samples is a precise method for defining the day of ovulation in common marmosets, the recording of core body temperature in this standard experimental setting proved not to be an appropriate method to monitor the reproductive cycle in this New World monkey species. 


\section{Appendix A}

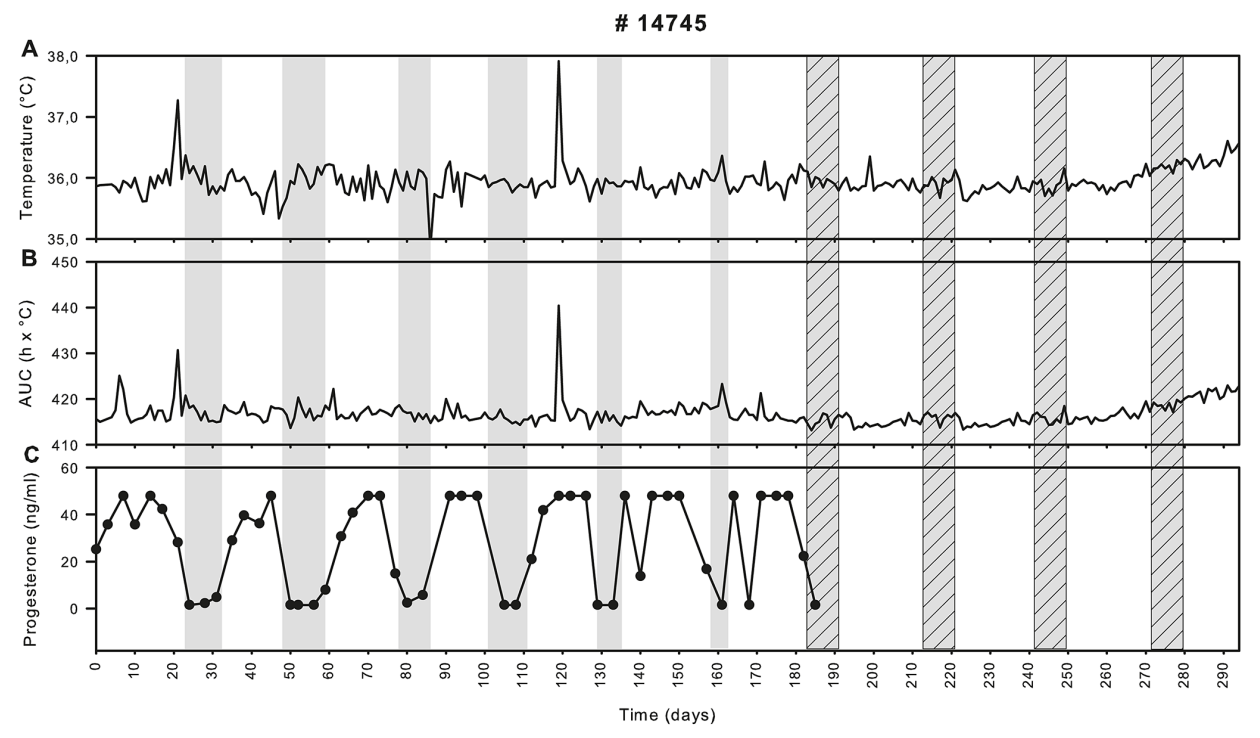

Figure A1. Diurnal variations in (a) minimal body temperature $\left(T_{\min }\right)$, (b) area under the curve (AUC) of nocturnal body temperature, and (c) serum progesterone concentrations for the complete study period (six progesterone data-based cycles followed by four extrapolated cycles) for animal \#14745. Follicular phases are highlighted in gray, and hatched boxes indicate the follicular phases of extrapolated cycles.

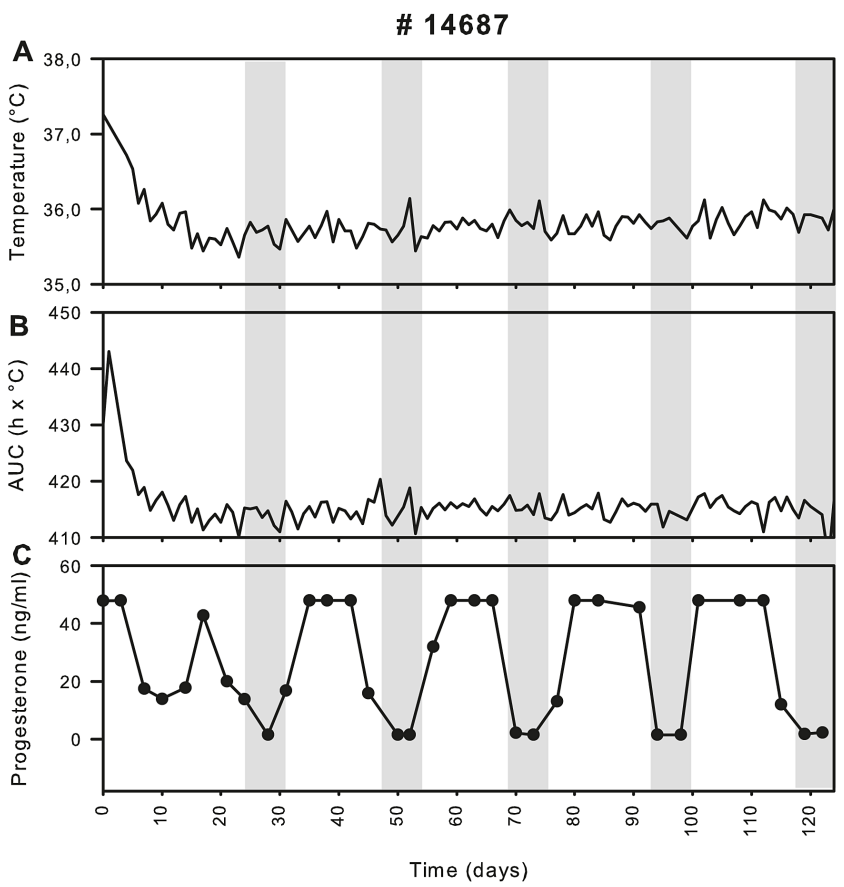

Figure A2. Diurnal variations in (a) $T_{\min }$, (b) AUC of nocturnal body temperature, and (c) serum progesterone concentrations for the complete study period (five cycles) for animal \#14687. Follicular phases are highlighted in gray. 
\# 14936
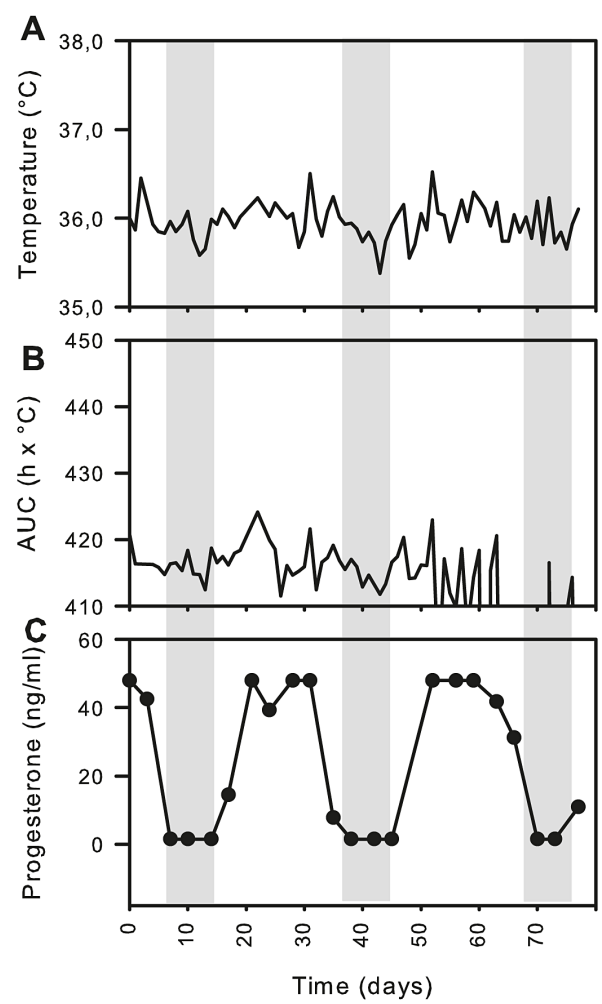

Figure A3. Diurnal variations in (a) $T_{\min }$, (b) AUC of nocturnal body temperature, and (c) progesterone concentrations for the complete study period ( 2.5 cycles) for animal \#14936. Follicular phases are highlighted in gray.
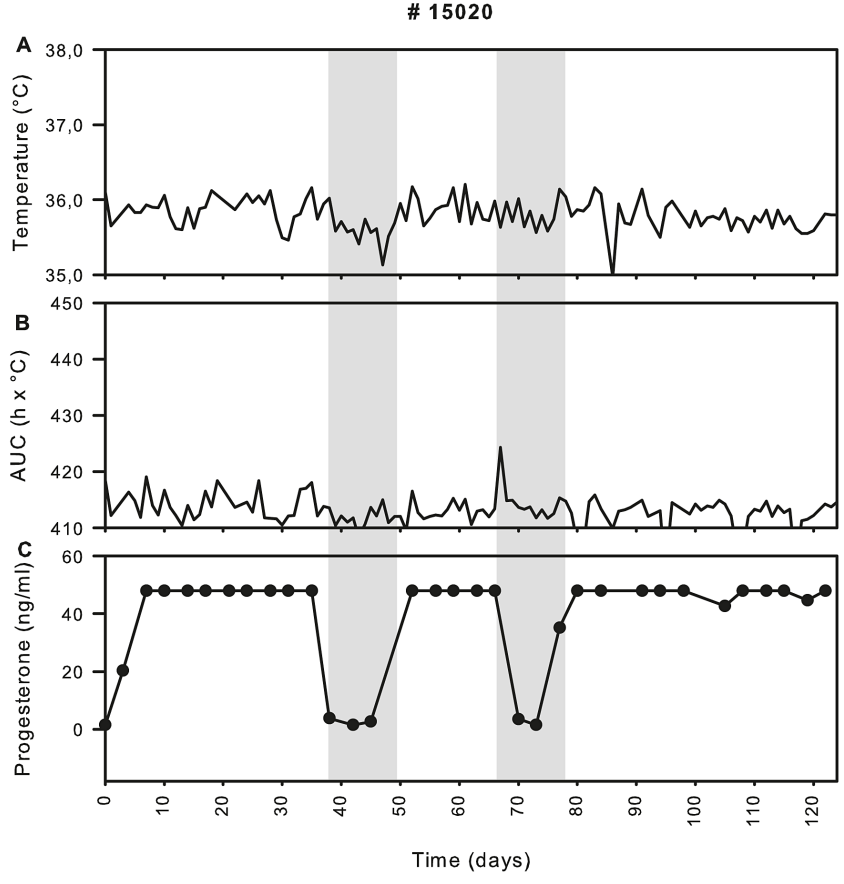

Figure A4. Diurnal variations in (a) minimal body temperature $\left(T_{\min }\right)$, (b) area under the curve (AUC) of nocturnal body temperature, and (c) serum progesterone concentrations for animal \#15020. Follicular phases are highlighted in gray. This animal was found to have an irregular reproductive cycle, with abnormally long elevated progesterone concentrations, and was therefore excluded from further analysis. 

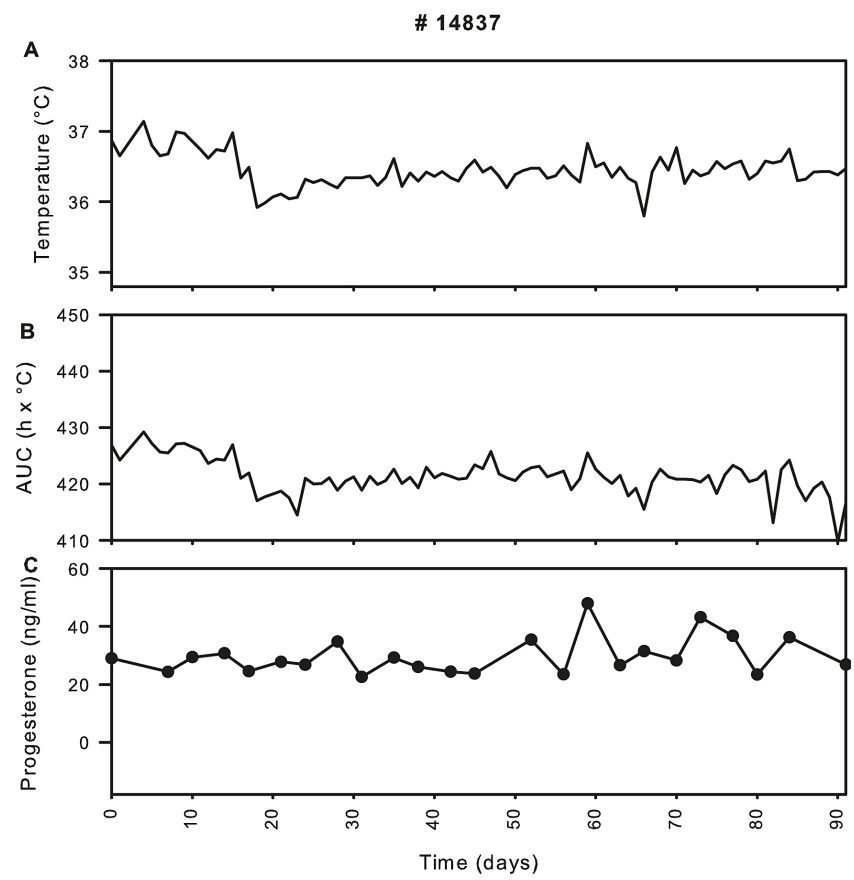

Figure A5. Diurnal variations in (a) minimal body temperature $\left(T_{\min }\right)$, (b) area under the curve (AUC) of nocturnal body temperature, and (c) serum progesterone concentrations for animal \#14837. This animal showed no clear reproductive cycles, with constantly elevated progesterone concentrations, and was therefore excluded from further analysis.

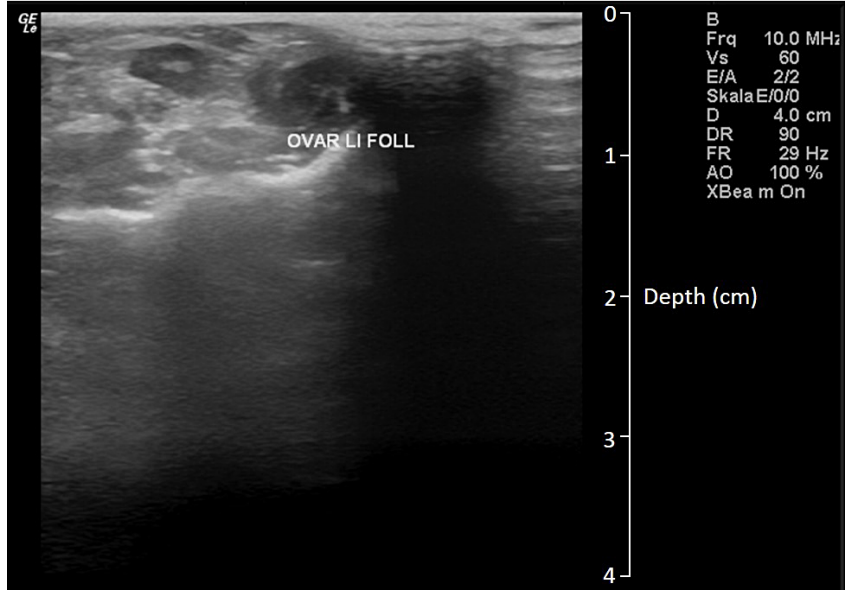

Figure A6. Representative ultrasound image (Frq: $10.0 \mathrm{MHz}$; depth $4.0 \mathrm{~cm}$ ) of the left ovary of animal \#14687 in the follicular phase (OVAR LI FOLL). Follicles are characterized by low echogenic (dark gray and black) round structures with defined margins.

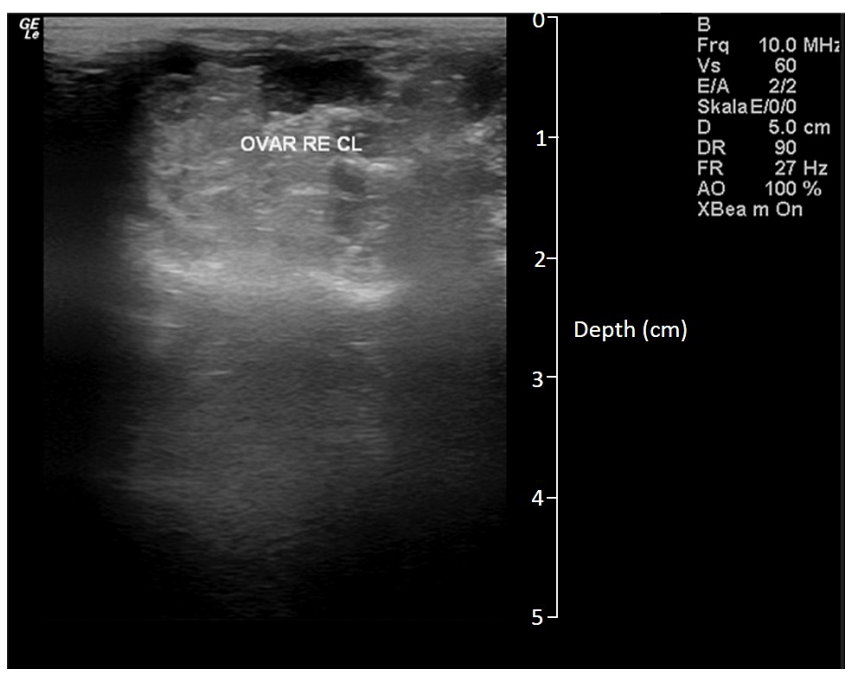

Figure A7. Representative ultrasound image (Frq: $10 \mathrm{MHz}$; depth $5.0 \mathrm{~cm}$ ) of the right ovary of animal \#14936 in the luteal phase (OVAR RE CL). The corpus luteum is characterized by a low echogenic (light gray) structure with indistinct demarcation. 
Acknowledgements. The authors would like to thank Sandra Leineweber, Olaf Rautz, Luise Krautheim, Janine Wülker and Julia Schell for taking care of the animals and Angelina Berenson, Nicole Umland and Thilo Speckmann for technical assistance as well as Michael Heistermann and Andrea Heistermann for technical support in analyzing the blood samples.

Edited by: S. Schlatt

Reviewed by: two anonymous referees

\section{References}

Baker, F. C. and Driver, H. S.: Circadian rhythms, sleep, and the menstrual cycle, Sleep Med, 8, 613-622, 2007.

Baker, F. C., Waner, J. I., Vieira, E. F., Taylor, S. R., Driver, H. S., and Mitchell, D.: Sleep and 24 hour body temperatures: a comparison in young men, naturally cycling women and women taking hormonal contraceptives, J. Physiol., 530, 565-574, 2001.

Balin, H. and Wan, L. S.: The significance of circadian rhythms in the search for the moment of ovulation in primates, Fert Steril, 19, 228-243, 1968.

Barger, L. K., Hoban-Higgins, T. M., and Fuller, C. A..: Assessment of circadian rhythms throughout the menstrual cycle of female rhesus monkeys, Am. J. Primatol., 70, 19-25, 2008.

Baum, M. J. and Bakker, J.: Roles of sex and gonadal steroids in mammalian pheromonal communication, Front Neuroendocrinol., 34, 268-284, 2013.

Cagnacci, A., Soldani, R., Laughlin, G. A., and Yen, S. S.: Modification of circadian body temperature rhythm during the luteal menstrual phase: role of melatonin, J. Appl. Physiol., 80, 25-29, 1996.

Cagnacci, A., Volpe, A., Paoletti, A. M., and Melis, G. B.: Regulation of the 24-hour rhythm of body temperature in menstrual cycles with spontaneous and gonadotropin-induced ovulation, Fertil. Steril., 68, 421-425, 1997.

Cagnacci, A., Arangino, S., Tuveri, F., Paoletti, A. M., and Volpe, A.: Regulation of the $24 \mathrm{~h}$ body temperature rhythm of women in luteal phase: role of gonadal steroids and prostaglandins, Chronobiol. Int., 19, 721-730, 2002.

Coyne, M. D., Kesick, C. M., Doherty, T. J., Kolka, M. A., and Stephenson, L. A.: Circadian rhythm changes in core temperature over the menstrual cycle: method for noninvasive monitoring, Am. J. Physiol. Regul. Integr. Comp. Physiol., 279, R1316R1320, 2000.

Dallmann, R., Steinlechner, S., von Hörsten, S., and Karl, T.: Stressinduced hyperthermia in the rat: comparison of classical and novel recording methods, Lab. Anim., 40, 186-193, 2006.

Dixson, A.: Effects of testosterone on the sternal cutaneous glands and genitalia of the male greater Galago (Galago crassicaudatus crassicaudatus), Folia Primatol., 26, 207-213, 1976.

Eikelboom, R.: Learned anticipatory rise in body temperature due to handling, Physiol. Behav., 37, 649-653, 1986.

Epple, G.: Olfactory communication in South American primates, Ann. NY Acad. Sci., 237, 261-278, 1974.

Forman, R. G., Chapman, M. C., and Steptoe, P. C..: The effect of endogenous progesterone on basal body temperature in stimulated ovarian cycles, Hum. Reprod., 2, 631-634, 1987.
French, J.A., Brewer, K. J., Schaffner, C. M., Shelly, J., HightowerMerritt, D., Smith, T. E., and Bell, S. M.: Urinary steroid and gonadotropin excretion across the reproductive cycle in female wied's black tufted-ear marmosets (Callithrix kuhli), Am. J. Primatol., 40, 231-245, 1996.

Gluckman, T. L., Walz, S. E., Schultz-Darken, N., and Bolton, I. D.: Cytologic assessment of the vaginal epithelium in the common marmoset (Callithrix jacchus): a preliminary new approach to reproductive screening, Contemp. Top. Lab. Anim. Sci., 43, 28-31, 2004.

Graham, C. E., Warner, H., Misener, J., Collins, D. C., and Preedy, J. R.: The association between basal body temperature, sexual swelling and urinary gonadal hormone levels in the menstrual cycle of the chimpanzee, J. Reprod. Fertil., 50, 23-28, 1977.

Hampl, R., Starka, L., and Jansky, L.: Steroids and thermogenesis, Physiol. Res., 55, 123-131, 2006.

Hanazawa, K., Mueller, T., Becker, T., Heistermann, M., Behr, R., and Sasaki, E.: Minimally invasive transabdominal collection of preimplantation embryos from the common marmoset monkey (Callithrix jacchus), Theriogenology, 78, 811-816. 2012.

Harding, R. D., Hulme, M. J., Lunn, S. F., Henderson, C., and Aitken, R. J.: Plasma progesterone levels throughout the ovarian cycle of the common marmoset (Callithrix jacchus), J. Med. Primatol., 11, 43-51, 1982.

Harlow, C. R., Gems, S., Hodges, J. K., and Hearn, J. P.: The relationship between plasma progesterone and the timing of ovulation and early embryonic development in the marmoset monkey (Callithrix jacchus), J. Zool., 201, 273-282, 1983.

Harlow, C. R., Hearn, J. P., and Hodges, J. K.: Ovulation in the marmoset monkey: endocrinology, prediction and detection, J. Endocrinol., 103, 17-24, 1984.

Hearn, J. P.: The common marmoset (Callithrix jacchus), in: Reproduction in new world primates: new models in medical science, edited by: Hearn, J. P., 181-215, MTP Press: Lancester, 1983.

Heistermann, M., Tari, S., and Hodges, J. K.: Measurement of faecal steroids for monitoring ovarian function in New World primates, Callitrichidae, J. Reprod. Fertil., 99, 243-251, 1993.

Hoffmann, K., Coolen, A., Schlumbohm, C., Meerlo, P., and Fuchs, E.: Remote long-term registrations of sleep-wake rhythms, core body temperature and activity in marmoset monkeys, Behav. Brain Res., 235, 113-123, 2012.

Lee, K. A.: Circadian temperature rhythms in relation to menstrual cycle phase, J. Biol. Rhythms, 3, 255-263, 1988.

Mansfield, K.: Marmoset models commonly used in biomedical research, Comp. Med., 53, 383-392, 2003.

Marshall, J.: Natural family planning, Lancet, 2, 1085-1086, 1976.

Marshall, J.: A field trial of the basal-body-temperature method of regulating births, Lancet, 2, 8-10, 1968.

Michael, R. P. and Zumpe, D.: A review of hormonal factors influencing the sexual and aggressive behavior of macaques, Am. J. Primatol., 30, 213-241, 1993.

Mustoe, A. C., Jensen, H. A., and French, J. A.: Describing ovarian cycles, pregnancy characteristics, and the use of contraception in female white-faced marmosets, Callithrix geoffroyi, Am. J. Primatol., 74, 1044-1053, 2012.

Nievergelt, C. and Pryce, C. R.: Monitoring and controlling reproduction in captive common marmosets on the basis of urinary oestrogen metabolites, Lab. Anim., 30, 162-170, 1996. 
Norris, S.: Basal body temperature in ovulation, Can. Med. Assoc. J., 67, 336-338, 1952.

Nunes, S., Brown, C., and French, J. A.: Variation in circulating and excreted estradiol associated with testicular activity in male marmosets, Am. J. Primatol., 56, 27-42, 2002.

Nyakudya, T. T., Fuller, A., Meyer, L. C., Maloney, S. K., and Mitchell, D.: Body temperature and physical activity correlates of the menstrual cycle in chacma baboons (Papio hamadryas ursinus), Am. J. Primatol., 74, 1143-1153, 2012.

Orsi, A., Rees, D., Andreini, I., Venturella, S., Cinelli, S., and Oberto, G.: Overview of the marmoset as a model in nonclinical development of pharmaceutical products, Regul. Toxicol. Pharmacol., 59, 19-27, 2011.

Petry, H. and Maier, J.: Radiotelemetrische Untersuchungen der Körpertemperatur von Weißbüscheläffchen (Callithrix jacchus), Z. Ernährungswiss., 29, 197-207, 1990.

Poole, S. and Stephenson, J. D.: Core temperature: some shortcomings of rectal temperature measurements, Physiol. Behav., 18, 203-205, 1977.

Schmelting, B., Corbach-Söhle, S., Kohlhause, S., Schlumbohm,C., Flügge, G., and Fuchs, E.: Agomelatine in the tree shrew model of depression: Effects on stress-induced nocturnal hyperthermia and hormonal status, Europ. Neuropsychopharmacol., 24, 437447, 2014.

Shille, V. M., Wing, A. E., Lasley, B. L., and Banks, J. A.: Excretion of radiolabeled estradiol in the cat (Felis catus, L): A preliminary report, Zoo Biology, 3, 201-209, 1984.

Sousa, M. B. and Ziegler, T. E.: Diurnal variation on the excretion patterns of fecal steroids in common marmoset (Callithrix jacchus) females, Am. J. Primatol., 46, 105-117, 1998. 't Hart, B. A., Abbott, D. H., Nakamura, K., and Fuchs, E.: The marmoset monkey: a multi-purpose preclinical and translational model of human biology and disease, Drug Discov. Today, 17, 1160-1165, 2012.

von Holst, D. and Eichmann, F.: Sex.specific regulation of marking behavior by sex hormones and conspecific scent in tree shrews (Tupaia belangeri), Physiol. Behav., 63, 157-164, 1998.

Ziegler, T. E. and Sousa, M. B.: Parent-daughter relationships and social controls on fertility in female common marmosets, Callithrix jacchus, Horm. Behav., 42, 356-367, 2002.

Ziegler, T. E., Sholl, S. A., Scheffler, G., Haggerty, M. A., and Lasley, B. L.: Excretion of estrone, estradiol, and progesterone in the urine and feces of the female cotton-top tamarin (Saguinus oedipus oedipus), Am. J. Primatol., 17, 185-195, 1989.

Ziegler, T. E., Scheffler, G., Wittwer, D. J., Schultz-Darken, N., Snowdon, C. T., and Abbott, D. H.: Metabolism of reproductive steroids during the ovarian cycle in two species of callitrichids, Saguinus oedipus and Callithrix jacchus, and estimation of the ovulatory period from fecal steroids, Biol. Reprod., 54, 91-99, 1996.

Zühlke, U. and Weinbauer, G.: The common marmoset (Callithrix jacchus) as a model in toxicology, Toxicol. Pathol., 31 Suppl, 123-127, 2003.

Zumpe, D., Bonsall, R. W., and Michael, R. P.: Some contrasting effects of surgical and "chemical" castration on the behavior of male cynomolgus monkeys (Macaca fascicularis), Am. J. Primatol., 26, 11-22, 1992. 